\title{
Índice de resiliencia de infraestructura de agua potable ante huracanes en ciudades costeras
}

\author{
Index of potable water infrastructure resilence facing \\ hurricanes in coastal cities
}

\author{
Anita Martínez Méndez ${ }^{1}$ \\ Oscar Frausto Martínez $z^{2}$ \\ Lourdes Castillo Villanueva ${ }^{3}$ \\ José Manuel Camacho Sanabria ${ }^{4}$ \\ Universidad de Quintana Roo, México
}

\begin{abstract}
Resumen
El objetivo de este trabajo fue determinar el índice de resiliencia de la infraestructura de agua potable de una ciudad costera ante huracanes. El modelo teórico utilizado se basa en las 4R de resiliencia y la variable de capacidad adaptativa. Como instrumentos para recopilación de información se utilizó un cuestionario a la población cuya aplicación fue por muestreo probabilístico. En el análisis de resultados se realizó con el software de Arcmap versión 10.3 con el fin de mostrar la diferencia espacial intraurbana. Posteriormente, se construyeron indicadores los cuales fueron estandarizados con base a su efecto positivo o negativo en la resiliencia, y con ello se determina el índice de la ciudad siendo de 0.69.
\end{abstract}

Palabras clave: crecimiento poblacional, demanda, zonas de riesgo, gestión integral del riesgo a desastre.

1 Maestra en Construcción. Instituto Tecnológico de Chetumal. Dirección: Boulevard Bahía s/n, colonia del Bosque, C.P. 77019 Chetumal, Quintana Roo. Correo electrónico: amm100983@gmail.com

2 Doctor en análisis e investigación espacial. Universidad Bauhaus Weimar, Alemania. Dirección: Avenida Andrés Quintana Roo, s/n, C.P. 77600 San Miguel de Cozumel, Quintana Roo, México. Correo electrónico: ofrausto@uqroo.edu.mx

3 Doctora en geografía. Universidad Autónoma de México. Dirección: Boulevard Bahía s/n, colonia del Bosque, C.P. 77019 Chetumal, Quintana Roo, México. Correo electrónico: loucasti@uqroo.edu.mx

4 Doctor en Ciencias Ambientales. Dirección: Boulevard Bahía s/n, colonia del Bosque, 77019 Chetumal, Quintana Roo, México Correo electrónico: jmanuelcs@live.com.mx

Este artículo corresponde a la ponencia presentada en el 35th Conference of Latin American Geographers realizada en San José, Costa Rica del 20 al 22 de mayo del 2018. 


\begin{abstract}
The aim of this research was to determine the index of potable water infrastructure resilence facing hurricanes in a coastal city. The theoretical model is based on the $4 \mathrm{R}$ resilence and the adaptive capacity variable. To gather the data, a questionnaire was applied to the population, as a probabilistic sampling. Arcmap v. 10.3 was used to analyze the results in order to show the interurban spatial difference. Afterwards, some indicators were created and standarized according to their positive or negative effect in the resilence, which helped us to determine the index of the city as 0.69 .
\end{abstract}

Keywords: population rise, request, risk areas, global management of disaster risk.

\title{
Introducción
}

El crecimiento demográfico, la urbanización y el aumento del consumo de agua en los hogares ha generado una alta demanda del servicio de agua potable, dotación apta para consumo humano, ampliaciones de los sistemas o, en su caso, la construcción de nuevos sistemas de abastecimiento, siendo retos que enfrenta la infraestructura de agua potable (Labaka et al, 2015 ).

Esta dinámica de crecimiento, se está concentrado en zonas costeras, que por sus características geográficas son vulnerables a los impactos del cambio climático, tales como son: huracanes, tormentas tropicales, lluvias atípicas, marejada de tormentas y erosión costera (Castillo y Velázquez, 2015; Yañez et al, 2010 y Azuz y Rivera, 2007; 2009). Estas tendencias demográficas y la necesidad del servicio vital, modifican consecutivamente los niveles de demanda y dotación, y esto a la vez genera transformaciones en los sistemas de infraestructura. Ilaya et al, (2015) señalan que cuando no se consideran las modificaciones de un sistema la capacidad y la influencia de las nuevas extensiones, se puede llegar a escenarios que tienden a reducir la capacidad de la red, amenazando a la cantidad y a la calidad del servicio, lo que está asociado directamente con la infraestructura.

A nivel nacional, para el año 2000 se tuvo una cobertura del servicio de agua potable de $85 \%$ y, en 2005 , de $88 \%$, aumentando en un $3 \%$; para el 2010 se logró un 89\% (INEGI, 1990; 2010), siendo los sectores más pobres y ubicados en zonas de riesgo los que más carecen del servicio (Domínguez, 2010). Esta situación hace evidente que los sistemas de infraestructuras no han sido planificados ni diseñados en sentido de las proyecciones de crecimiento poblacional y urbano, y de continuar bajo los mismos esquemas de planificación y diseño, las problemáticas que actualmente se presentan aumentarán. Es por ello, que se hace prioritario la 
Anita Martínez Méndez, Oscar Frausto Martínez, Lourdes Castillo Villanueva, José Manuel Camacho Sanabria. Índice de resiliencia de infraestructura de agua potable ante huracanes en ciudades costeras

creación de infraestructuras bajo enfoques que sean capaces de responder a las situaciones generadas por la naturaleza y la acción humana.

En este sentido, en el presente trabajo se mostrará la construcción del índice de resiliencia de la infraestructura de agua potable ante huracanes en un sistema urbano costero, que permitirá contextualizar la resiliencia, mediante indicadores de influencia positiva y negativa. Se inicia dando a conocer la ubicación de la zona de estudio, se contextualiza las características generales del sistema, se muestra la teoría que fundamente el trabajo, el marco metodológico, se exponen los resultados por zona y a escala ciudad y, por último, se realiza la discusión de resultados.

\section{Área de estudio}

La ciudad de Chetumal se ubica en el extremo suroriental de México, en el Caribe, entre los paralelos $18^{\circ} 33^{\prime} 46^{\prime \prime}$, y $18^{\circ} 29^{\prime} 40^{\prime \prime}$, y en los meridianos $88^{\circ} 21^{\prime} 57^{\prime \prime}$ y $88^{\circ} 16^{\prime} 45^{\prime}$ "; con una superficie de 18158 hectáreas (CAPA, 2015), es una ciudad costera con 151243 habitantes que se dedican al desarrollo de actividades terciarias, principalmente a la administración pública y el comercio (INEGI, 2010). 
Figura 1. Ubicación geográfica del área de estudio

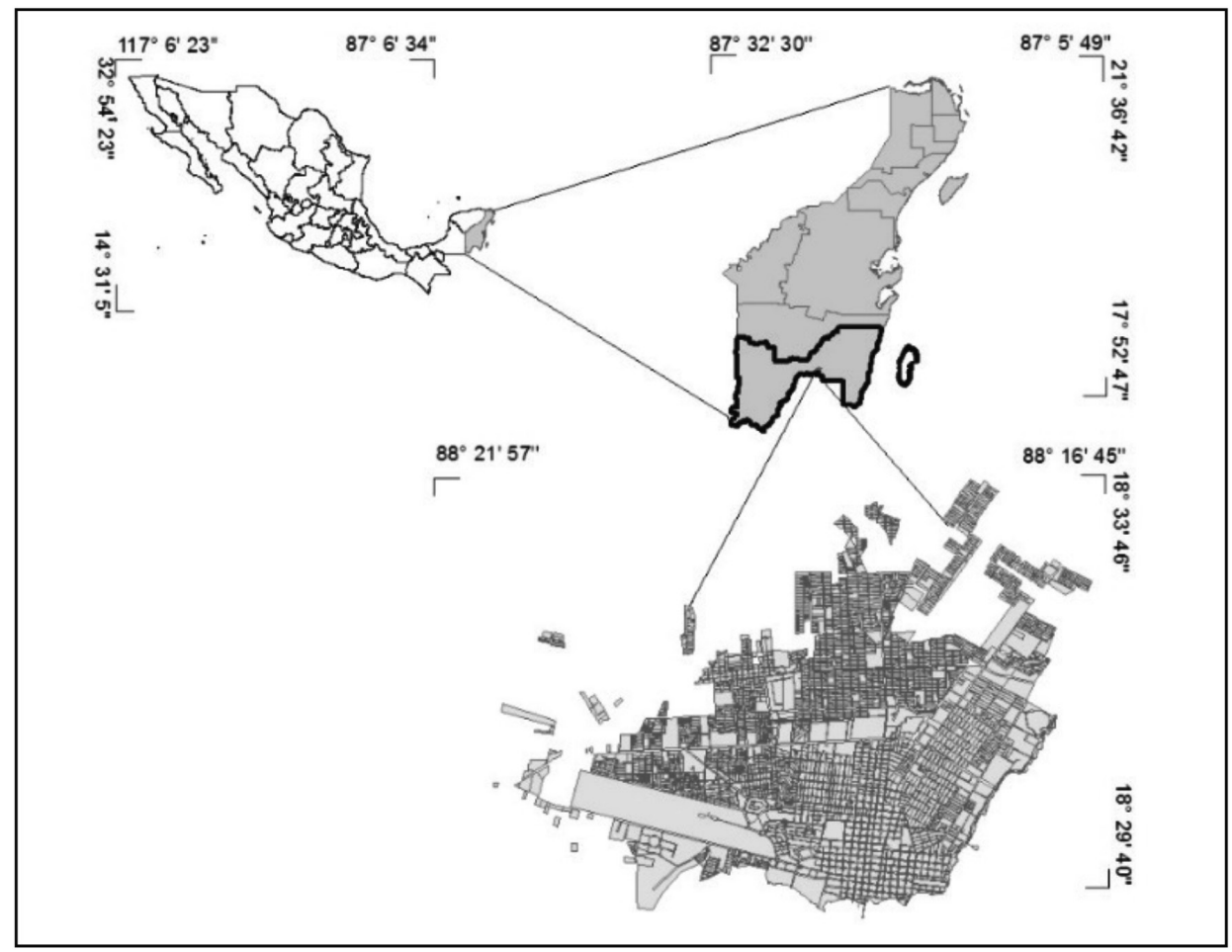

Fuente: elaboración propia con base a INEGI (2016).

\section{Características generales del sistema de infraestructura de agua potable}

El sistema de infraestructura urbana de agua potable ha tenido un crecimiento parcialmente paralelo a la dinámica de desarrollo demográfico y urbano. En el año 2000 la ciudad contaba con 121602 habitantes, que vivían en 30003 viviendas de las cuales el 94\% contaba con el servicio de agua potable; para el año 2007, se registra un crecimiento de $12.5 \%$ en población, $7 \%$ en viviendas y un abastecimiento del 97\%. En el año 2010, la tendencia de desarrollo continuo en aumento, siendo de $10 \%$ en habitantes, $30 \%$ en viviendas y se presentaba un $96 \%$ de dotación del servicio. Finalmente, en el año 2015, se registra un aumento de 11\% de habitantes, $27 \%$ en viviendas y el abastecimiento fue del $95 \%$ (figura 2 ). 
Anita Martínez Méndez, Oscar Frausto Martínez, Lourdes Castillo Villanueva, José Manuel Camacho Sanabria. Índice de resiliencia de infraestructura de agua potable ante huracanes en ciudades costeras

Figura 2. Crecimiento urbano y de servicio
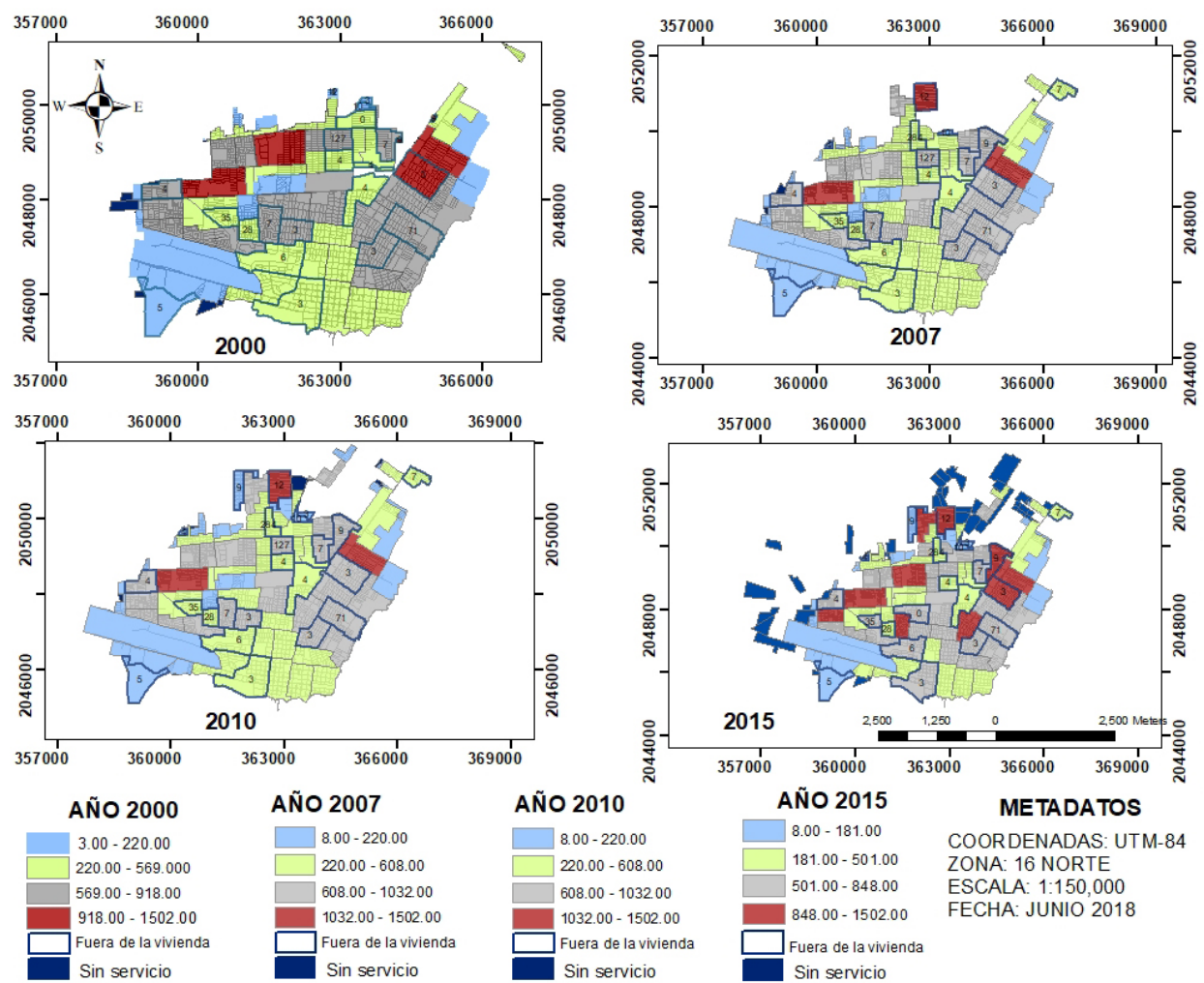

AÑO 2007

AÑO 2010

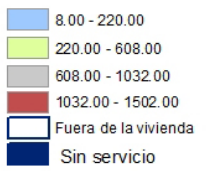

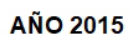

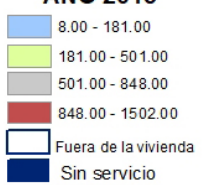

METADATOS

COORDENADAS: UTM- 84 ZONA: 16 NORTE

ESCALA: $1: 150,000$

FECHA: JUNIO 2018

Fuente: elaboración con base a datos de INEGI, 2000; 2007; 2010; 2016; Comisión de agua potable y alcantarillado, 2016.

La traza urbana se ha ido expandido al norte y noreste de la ciudad, donde se encuentran localizado el ecosistema del manglar y, además, parte de ese desarrollo ha sido bajo escalas informales, lo que ha modificado los usos de suelo, las características naturales de los ecosistemas e inconsistencias en la infraestructura de agua potable, como son ampliaciones no planificadas de la red principal y la construcción en zonas de riesgo.

De modo que la construcción de la infraestructura del sistema se está dando en zonas donde su desarrollo, funcionamiento e interdependencia con otros sistemas estará condicionado. Si bien, es cierto que está directamente asociado al desarrollo poblacional, no deja de poseer características que son exclusivas del sistema. Esto muestra la falta de planificación o en su caso la proyección bajo esquemas no acordes al contexto de la ciudad. 
Al respecto Martín y Justo (2015) señalan que este proceso puede deberse a diversas causas, como una débil gobernabilidad, ineficacia de los marcos normativos, falta de capacidades institucionales, de medios materiales y humanos adecuados, fragmentación del poder decisorio y la falta de participación ciudadana.

\section{Resiliencia en la infraestructura}

En el contexto urbano el concepto de resiliencia se orienta principalmente a los problemas asociados al cambio climático como los son los huracanes, lluvias atípicas, inundaciones, y heladas por mencionar algunos (Vázquez et al., 2016; Leichenko, 2011; Zhou et al, 2010). Los avances en la investigación han generado una diversidad de definiciones con varios enfoques como el social, comunitario, local, en el contexto de desastres, político, urbano, económico y en la infraestructura.

Sin embrago, existen definiciones que han servido como base y continúan siendo utilizadas por organismos, instituciones e investigadores. Dentro de ellas se encuentra lo planteado por Holling (1973), quien define resiliencia desde la perspectiva ecológica como "una medida de la persistencia de los sistemas y de su capacidad para absorber los cambios y las perturbaciones y mantener las mismas relaciones entre poblaciones o estados variables". Walker et al. (2004), plantean un enfoque similar definiéndola como la capacidad de un sistema para absorber perturbaciones y reorganizarse mientras experimenta cambios a fin de conservar esencialmente la misma función, estructura, identidad y retroalimentación.

El mismo Holling, en 1996, realizó un contraste de la resiliencia en sentido de ingeniería y ecológico. Señalando que la resiliencia en ingeniería es determinada como la estabilidad cerca de un estado estable, donde la resistencia a la perturbación y la velocidad de retorno al equilibrio se utilizan para medir la propiedad (O'Neill et al., 1986; Pimm, 1984; Tilman y Downing, 1994 citados en Holling, 1996). Al respecto, Folke (2010) señala que este tipo de resiliencia se centra en el comportamiento de un equilibrio estable y la velocidad en la que un sistema se aproxima a su estado estacionario después de una perturbación, es decir, la velocidad de retorno al equilibrio.

Estas definiciones se centran en los temas de estabilidad de un sistema, capacidad de absorber perturbaciones, de recuperarse y en el tiempo 
Anita Martínez Méndez, Oscar Frausto Martínez, Lourdes Castillo Villanueva, José Manuel Camacho Sanabria. Índice de resiliencia de infraestructura de agua potable ante huracanes en ciudades costeras

de retorno a su equilibrio. Desde esta perspectiva, para el estudio de la resiliencia se ha considerado como unidad de análisis a los sistemas socio-ecológicos definiéndolos como una compleja estructura que puede ser analizada considerando el subsistema social y el subsistema ecológico (sociedad-ambiente). El primero se conforma por instituciones políticas, económicas y sociales, espiritualidad, artes y cultura; el segundo con los ecosistemas minerales, hidrología, clima, procesos físicos, químicos y biológicos de la biosfera (Raskin, 2006, citado en Castillo y Velázquez, 2015).

Para propósitos de este trabajo se considera la conceptualización de resiliencia con enfoque en los sistemas de infraestructura. La resiliencia de un sistema de infraestructura es considerada como; la capacidad de un sistema para ajustar su funcionamiento antes, durante o después de los cambios y las perturbaciones, de modo que pueda continuar funcionando según sea necesario después de una interrupción, y en presencia de tensiones continuas (Dekker et al, 2008 citado en Tamvakis y Xenidis 2013). Al respecto, Gays (2016) señala que una infraestructura resiliente "no necesariamente es aquella que nunca falla, sino aquella que, habiendo sufrido un evento (natural o antropogénico) es capaz de sostener un nivel mínimo de servicio y recuperar su funcionamiento original en un tiempo corto y con inversión moderada".

Por otra parte, Windle et al., (2011) señalan que la resiliencia requiere su operacionalización. Al respecto, diversos autores como Ahern et al., 2006; Tonni, 2000; Bruneau et al, 2003; O’Rourke, 2007;2001; Vugrin et al., 2010; Youn et al., 2011; Henry et al., 2012; Barker et al., 2013; Frankenberg et al, 2013; Vázquez et al., 2016; han realizado propuestas para cuantificar la resiliencia en diversos tipos de infraestructura, considerando variables como confiabilidad, demanda del servicio de agua potable, calidad del agua, restauración del sistema, capacidad de recuperación, tiempo de recuperación, el impacto positivo de un sistema, el estado normal del sistema y después de la perturbación, costos de recuperación, número de conexiones, viviendas con agua potable, viviendas sin agua potable, redundancia, robustez, recursos y conservación de energía, entre otros.

Para el objetivo de esta investigación se retomara lo propuesto por Gays, 2016; Labaka et al. (2015); Bruneau 2003 y O’Rourke, 2007; considerando las variables de robustez, redundancia, recursos, tiempo de recuperación y capacidad adaptativa (figura 3). En esta última variable, si bien, 
los autores la manejan refiriéndose específicamente a las características de las partes físicas del sistema y al tiempo en que este logra su adaptación sin realizar ninguna actividad de recuperación; sin embargo, esa capacidad del sistema en caso de desastres también depende de otros factores que tienen influencia desde el diseño, construcción, desarrollo y operación. La resiliencia en la infraestructura no puede recaer únicamente en los sistemas físicos, como tampoco de quien las construye. Al respecto Walker et al., 2006 (citado en Castillo y Velázquez, 2015), señalan que la adaptación de un sistema socio-ecológico está determinada en primera instancia por la cantidad absoluta y relativa de todas las formas de capital: social, humana, financiera, física, natural, político y por el sistema de instituciones y gobernanza.

Figura 3. Modelo teórico

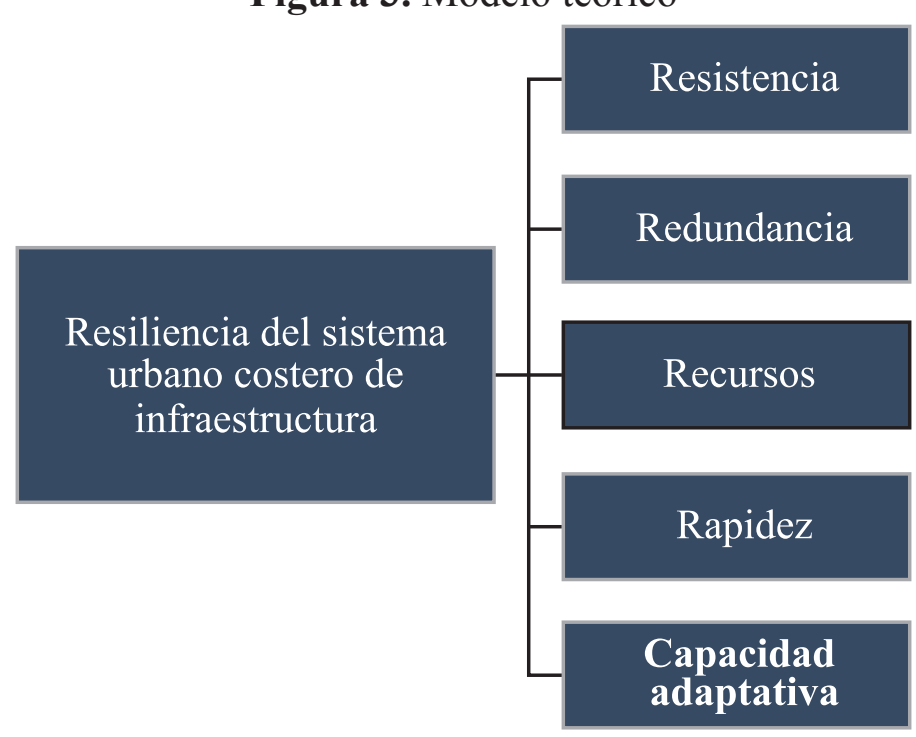

Fuente: elaborado en base a Gays, 2016; Lansey (2012); Yazdani et al. (2011; Vugrin et al., 2010; O’Rourke, 2007 y Bruneau 2003.

Así, los elementos centrales de la resiliencia del sistema urbano costero son:

- Resistencia: La capacidad de un sistema de no colapsarse totalmente ante una falla, sino conservar un mínimo necesario de funcionamiento. 
Anita Martínez Méndez, Oscar Frausto Martínez, Lourdes Castillo Villanueva, José Manuel Camacho Sanabria. Índice de resiliencia de infraestructura de agua potable ante huracanes en ciudades costeras

- Redundancia: el sistema debe poseer suficientes redundancias, para evitar que haya cuellos de botella o elementos que al fallar causen la falla completa del sistema.

- Recursos: Se refiere no únicamente a tener recursos para atender una emergencia, tales como repuestos y personal, sino también al ingenio para improvisar soluciones temporales que sostengan el funcionamiento del sistema.

- Rapidez: se refiere al tiempo de recuperación del sistema después de haber sufrido una falla provocada por un evento.

- $\quad$ Capacidad adaptativa: se refiere a la fortaleza de los sistemas de infraestructura adaptarse a los cambios que genere un evento perturbador.

\section{Marco Metodológico}

\section{Elaboración del cuestionario}

El instrumento se estructuro en tres secciones: la primera corresponde a datos generales del entrevistado y de la zona, la dos se integró por tres preguntas referentes a los antecedentes considerando las variable: motivo de vivir en la zona, años de vivir en la zona y experiencia de huracán, la última sección se conformó de 22 preguntas relacionadas a las características del sistema de agua potable, siendo estructuradas de acuerdo a la incidencia que tienen en la robustez, redundancia, recursos, rapidez y capacidad adaptativa del sistema.

\section{Cálculo y distribución de la muestra}

La población objetivo considerada para el cálculo de la muestra, fueron habitantes de18 años y más. La unidad de muestreo fue el total de hogares tomado del censo de población y vivienda del 2010. El cálculo se realizó mediante la siguiente formula:

$$
n=\frac{k^{2} * q * d}{r^{2} * p *(1-t)}
$$

Los parámetros utilizados (d, r y t) fueron tomados de la encuesta intercensal 2015 realizada por el INEGI a nivel municipal. Esto considerando que Chetumal representa el 61\% del total del municipio de Othón P. Blanco. 
Obteniéndose una muestra total de 238 hogares; por razones de redondeo al número inmediato superior la muestra final quedo de 292 hogares.

El marco muestral utilizado fue el plano urbano de la ciudad de Chetumal, que está conformado por 131 áreas geoestadísticas básicas (ageb). Se clasificó con base a la variable "peligro ante huracanes" especificada en el Altas de Riesgo de la ciudad de Chetumal (2011), en tres estratos (tabla 1): riesgo muy alto, alto y medio.

Tabla 1. Distribución de muestra en estratos

\begin{tabular}{|l|c|c|c|c|}
\hline \multicolumn{1}{|c|}{ ESTRATO } & $\begin{array}{c}\text { No. DE } \\
\text { AGEBS }\end{array}$ & $\begin{array}{c}\text { TOTAL } \\
\text { HOGARES }\end{array}$ & $\begin{array}{c}\text { TOTAL } \\
\text { HOGARES } \\
\text { MUESTRA }\end{array}$ & $\begin{array}{c}\text { HOGARES } \\
\text { MUESTRA } \\
\text { AJUSTADO }\end{array}$ \\
\hline 1. Riesgo muy alto & 52 & 26502 & 139 & 169 \\
\hline 2. Riesgo alto & 34 & 16448 & 87 & 103 \\
\hline 3. Riesgo medio & 5 & 2243 & 12 & 20 \\
\hline TOTALES & $\mathbf{9 1}$ & $\mathbf{4 5 1 9 3}$ & $\mathbf{2 3 8}$ & $\mathbf{2 9 2}$ \\
\hline
\end{tabular}

Fuente: Elaborado en base a INEGI (2010).

El proceso de distribución de la muestra se realizó de la siguiente manera:

- $\quad$ Selección de ageb. Para ello se consideró la variable hogares habitados, obteniéndose 91 de las 131. Las restantes se omitieron toda vez que no cuentan con hogares habitados o en su caso corresponden a zonas industriales, infraestructura urbana y zonas de área verde.

- Distribución de muestra por ageb: se llevó a cabo por afijación proporcional con base los hogares, considerando que en una vivienda puede existir más de un hogar.

- Distribución de la muestra en manzanas: en esta fase se tuvieron las siguientes consideraciones: seleccionar una manzana en el AGEB, si correspondía encuestar de uno a dos hogares; tres manzanas si se requería entrevistar de tres a cinco hogares y más de tres manzanas cuando el número de hogares superaba los cinco, teniendo en cuenta que por manzana se aplicaran dos cuestionarios. Éstas fueron seleccionadas utilizando como instrumento la tabla de números aleatorios, por medio del procedimiento de selección aleatoria simple. 
Anita Martínez Méndez, Oscar Frausto Martínez, Lourdes Castillo Villanueva, José Manuel Camacho Sanabria. Índice de resiliencia de infraestructura de agua potable ante huracanes en ciudades costeras

- $\quad$ Selección de hogares. Este procedimiento fue por medio del muestreo aleatorio simple y se realizó en campo al momento de la aplicación del cuestionario, considerando únicamente las manzanas previamente elegidas y con la limitante de no encuestar dos hogares en la misma calle.

\section{Aplicación del cuestionario}

El cuestionario fue aplicado en el mes de marzo de 2018, en horarios de 10:00 a 13:00 y de 16:00 a 17:00 horas, de lunes a viernes, siendo horarios más aptos para la participación de la población de la zona de estudio.

\section{Construcción de índice de resiliencia}

Esta fase se llevó a cabo mediante los siguientes pasos:

- Identificación de indicadores: se realizó con base en: revisión bibliográfica de diversas propuestas de indicadores asociados al tema de investigación (Frausto et al, 2016; INEGI, 2016; IMTA, 2015; Moreno et al, 2015; PRODUS-UCR, 2014; Sánchez at al, 2015; Plan nacional de infraestructura 2014-2018; Programa Nacional Hídrico 2014-2018; CONAGUA-IMTA, 2014; USAID; 2013; BID, 2011; Cutter et al, 2003; 2008; Tierney, 2009; Carreño et al, 2005); y con la percepción de los usuarios sobre las problemáticas, potencialidades y líneas de oportunidad del sistema en relación a las afectaciones de huracán.

- Agrupación en componentes: Los indicadores se agruparon en cuatro elementos (tabla 2) que fueron establecidos en el modelo teórico propuesto (robustez, redundancia, recursos, rapidez y capacidad adaptativa) de acuerdo a la influencia positiva o negativa en la resiliencia.

Tabla 2. Indicadores por componente

\begin{tabular}{|l|c|}
\hline \multicolumn{1}{|c|}{ COMPONENTE } & INDICADORES \\
\hline Resistencia & 10 \\
\hline Redundancia & 8 \\
\hline Recursos & 4 \\
\hline Rapidez & 1 \\
\hline Capacidad adaptativa & 8 \\
\hline & $\mathbf{3 1}$ \\
\hline
\end{tabular}

Fuente: elaboración propia 
- $\quad$ Normalización de datos. En este proceso primero se transformaron los indicadores simples a medidas descriptivas (porcentajes, promedio, moda, rango), posteriormente se realizó la normalización con la técnica directa Min-Max.

- Ponderación. Esta realizó con base a la revisión bibliográfica, consulta de expertos (empresas privadas) y servidores públicos (CONAGUA, CAPA). Consistió en dar un valor a cada indicador simple de los componentes (ver anexo 1) y a cada componente o indicador compuesto (tabla 3 ).

Tabla 3. Ponderación a nivel componente

\begin{tabular}{|l|c|}
\hline \multicolumn{1}{|c|}{ COMPONENTE } & PONDERACIÓN \\
\hline Resistencia & 0.3 \\
\hline Redundancia & 0.3 \\
\hline Recursos & 0.2 \\
\hline Rapidez & 0.1 \\
\hline Capacidad adaptativa & 0.1 \\
\hline
\end{tabular}

Fuente: elaboración propia

- Agregación a nivel indicador. Este procedimiento se llevó a cabo en los tres estratos implementando el método de agregación multiplicativa en los 31 indicadores. Obteniendo con la sumatoria un indicador compuesto por cada componente.

- $\quad$ Agregación a nivel componente. Se aplicó por segunda vez el método de agregación multiplicativa, obtenido un índice por cada zona y a nivel ciudad.

\section{Resultados}

La ciudad de Chetumal se abastece de agua potable por medio de pozos profundos de agua subterránea, distribuidos espacialmente en tres zonas de captación ubicadas al sureste del municipio de Othón P. Blanco. La primera se localiza en la localidad de Xul-Ha, al sur de la laguna de Bacalar. La segunda y tercera zona se encuentra en la localidad Jesús González Ortega, en la en la carretera Chetumal-Escárcega. Las tres zonas forman parte del municipio de Othón P. Blanco (Figura 4). 
Anita Martínez Méndez, Oscar Frausto Martínez, Lourdes Castillo Villanueva, José Manuel Camacho Sanabria. Índice de resiliencia de infraestructura de agua potable ante huracanes en ciudades costeras

Figura 4. Sistema de abastecimiento de agua potable, Chetumal Quintana Roo, México

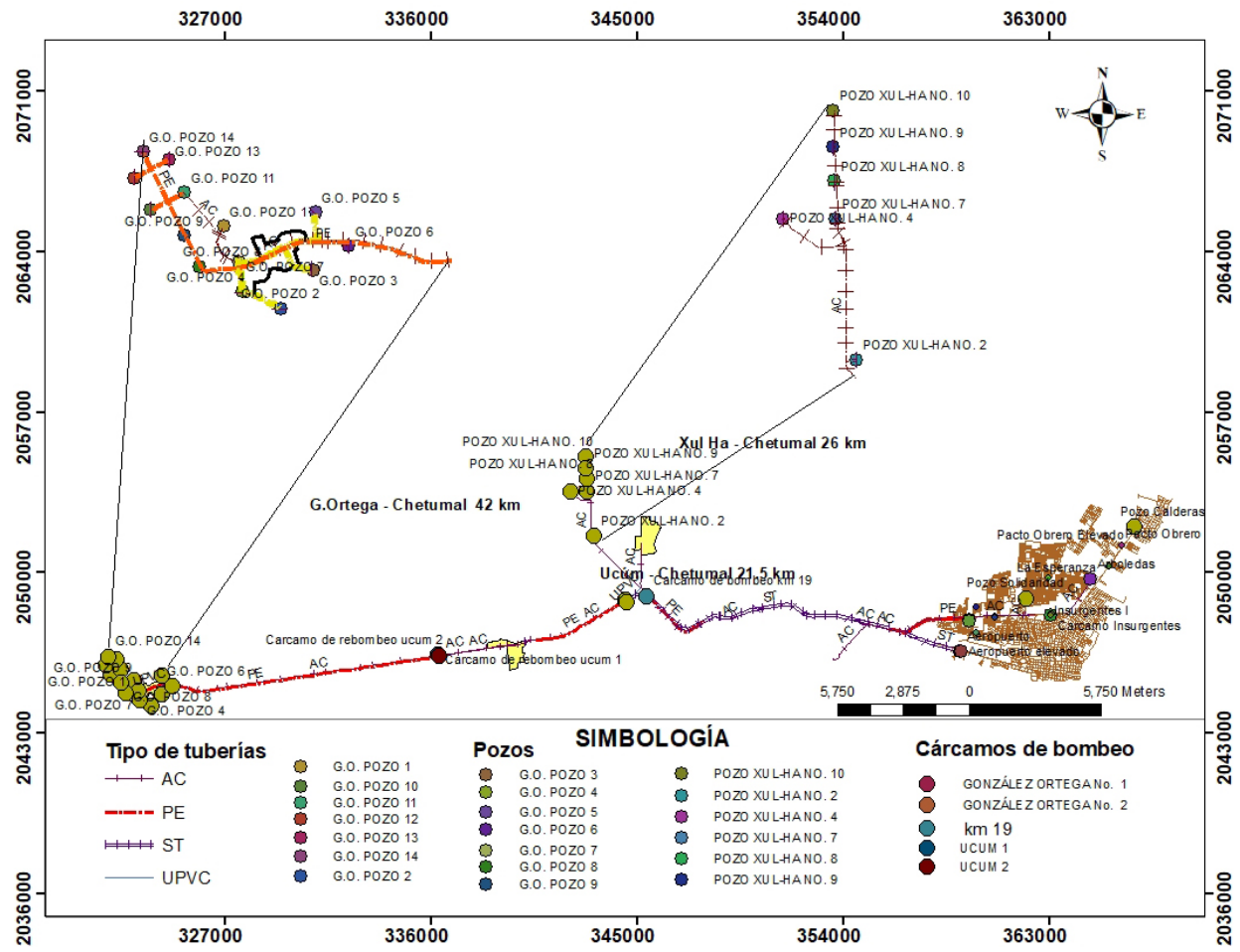

Fuente: Elaboración con base a la INEGI, 2016; CONAGUA, 2015; CAPA, 2015.

La primera zona se ubica en la localidad de Xul-ha a $26 \mathrm{~km}$ de la ciudad de Chetumal, inicio su operación en el año 1964, teniendo 53 años de funcionando. Está formada por diez pozos de captación de los cuales seis se encuentran en operación (P2, P4, P7, P8, P9, P10). Cuenta con una caseta de operación ubicada aproximadamente a $3.2 \mathrm{~km}$ de la localidad de Xul-Ha, descarga actualmente en un tanque de cambio de régimen (TCR), con un caudal de 140 1/s, las cuales operan con energía eléctrica de un transformador de $112.5 \mathrm{kva}$. La infraestructura que comprende la red de captación, está estructurada por tubería de 8", 10", 12" y 14" de asbesto cemento; su producción diaria es de 140 litros por segundo (1/s).

La segunda zona corresponde al sistema de captación de González Ortega I, este se creó en el año 1983, a la fecha tiene 34 años en funcionamiento, se localiza a $42 \mathrm{~km}$ de la ciudad de Chetumal; está formada por 
siete pozos de captación, los cuales se encuentran operando. Cuenta con una caseta de operación ubicada aproximadamente a $650 \mathrm{~m}$ del acceso de la localidad, y un cárcamo de bombeo de $70 \mathrm{~m}^{3}$ de capacidad, su operación es a base de energía eléctrica de un transformador de $300 \mathrm{kva}$. La infraestructura del sistema se conforma de tubería de 8" de PVC y de 6" de asbesto cemento. Su producción diaria es de 220 (1/s).

En esta misma zona se ubica la tercera zona la cual está conformada por siete pozos de captación de agua subterránea enumerados de manera consecutiva con respecto a la segunda (P8, P9, P10, P11, P12, P13, P14), estos se encuentran operando adecuadamente. Esta zona cuenta con una caseta de operación ubicada a $650 \mathrm{~m}$ de la localidad, y un cárcamo de bombeo de capacidad de $252 \mathrm{~m}^{3}$, su operación es a base de energía eléctrica de un transformador de $225 \mathrm{kva}$. La infraestructura se conforma por tuberías de 8 " de polietileno de alta densidad (PAD) y de asbesto cemento; la capacidad de producción de esta zona es de $240 \mathrm{l} / \mathrm{s}$, la cual es conducida a través de una red de 20 " con tubería de polietileno de alta densidad hacia el cárcamo.

La zona de captación Tanque de Cambio de Régimen (TCR) se encuentra a $21.5 \mathrm{~km}$ de la ciudad de Chetumal, se conforma por dos pozos de captación de aguas subterráneas, el primero inicio su funcionamiento en el año 2008 y el segundo en el 2011. Tiene una capacidad de $49.61 \mathrm{~m}^{3}$. En esta zona se reciben el agua desinfectada y mediante el TCR se realiza la conducción de agua potable por medio de gravedad con tubería de acero y de fibrocemento de 36", en el trayecto se alimentan a seis localidades, a los predios que se encuentran localizados en el tramo del recorrido del flujo y a cinco cárcamos de la ciudad de Chetumal (Aeropuerto, Bachilleres, Insurgentes, Solidaridad y Arboledas).

Para la conducción a los cinco cárcamos se realiza por medio de tuberías con diámetros de 14", 18", 20" y 24" de polietileno de alta densidad. La primera línea de distribución con tubería de 14" va al cárcamo aeropuerto ubicado al sur, la segunda con tubería de diámetro de 24 " que es conducida al cárcamo bachilleres localizado al este, la tercera con tubería de 20 " y 18 " que se conduce al cárcamo Solidaridad situado espacialmente al norte de la ciudad, y continua con la misma tubería hacia el cárcamo insurgentes ubicado al este y al arboledas ubicado al noroeste de la ciudad. 
Anita Martínez Méndez, Oscar Frausto Martínez, Lourdes Castillo Villanueva, José Manuel Camacho Sanabria. Índice de resiliencia de infraestructura de agua potable ante huracanes en ciudades costeras

\section{Índice de resiliencia del sistema urbano costero de infraestruc- tura de agua potable}

Se obtuvieron indicadores por componente de robustez, redundancia, recursos, rapidez y capacidad adaptativa, así como el índice de resiliencia del sistema urbano costero de infraestructura de agua potable desglosados de acuerdo a la clasificación de estrato peligro muy alto, alto y medio (Tabla 4). Los factores obtenidos oscilan entre cero y uno, donde el valor más cercano al máximo representa la posibilidad de una resiliencia muy alta y los próximos al valor mínimo indican la probabilidad de una resiliencia baja en el sistema.

Tabla 4. Indicador por estrato y componente

\begin{tabular}{|l|c|c|c|}
\hline \multirow{2}{*}{$\begin{array}{c}\text { INDICADOR POR } \\
\text { COMPONENTE }\end{array}$} & $\begin{array}{c}\text { PELIGRO MUY } \\
\text { ALTO }\end{array}$ & PELIGRO ALTO & $\begin{array}{c}\text { PELIGRO } \\
\text { MEDIO }\end{array}$ \\
\cline { 2 - 4 } & 0.56 & 0.52 & 0.58 \\
\hline Robustez & 0.83 & 0.87 & 0.81 \\
\hline Redundancia & 0.6 & 0.82 & 0.35 \\
\hline Rapidez & 0.97 & 0.99 & 0.86 \\
\hline Capacidad adaptativa & 0.72 & 0.64 & 0.5 \\
\hline
\end{tabular}

Fuente: elaboración propia

El competente correspondiente a robustez sus valores oscilaron entre 0.52 y 0.58 ; ubicando a las tres zonas en una resiliencia media, esto indica un resistencia media del sistema ante un evento perturbador (figura 5).

La redundancia que presento el sistema de agua potable, fue superior a la de la robustez en las tres zonas los cuales estuvieron entre 0.81 y 0.87 . De acuerdo a los niveles de resiliencia propuestas en el presente trabajo, el componente se ubica en la escala de resiliencia muy alta (figura 6). Esto indica que el sistema cuenta con alternativas de solución en caso de un evento o incluso equipos que puedan sustituir a los que en su caso fallen. Posteriormente se muestra el componente de recursos (figura7). Sus valores obtenidos se encontraron entre 0.35 y 0.82 ; detectándose que el valor menor se presenta en la zona correspondiente a peligro medio, esto indica que en esa área la inversión en la mejora del sistema y mitigación ante eventos perturbadores no se realiza con frecuencia, o incluso no se atienden 
Anita Martínez Méndez, Oscar Frausto Martínez, Lourdes Castillo Villanueva, José Manuel Camacho Sanabria. Index of potable water infrastructure resilence facing hurricanes in coastal cities

\section{Figura 5. Indicador robustez}

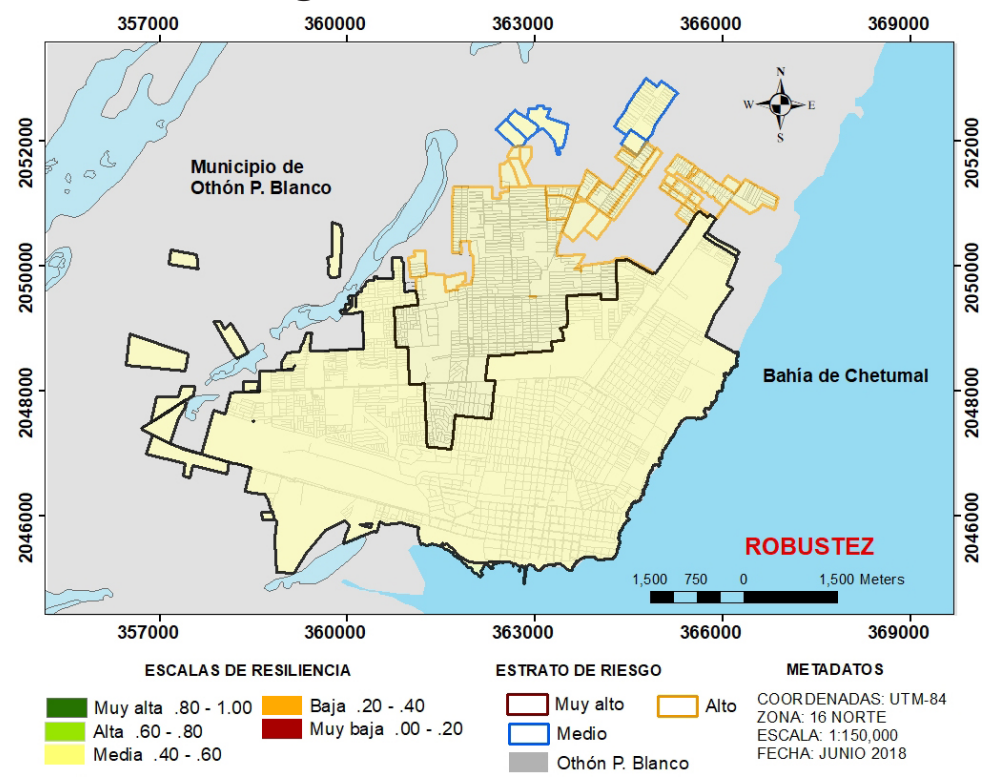

Fuente: elaboración propia

Figura 6. Indicador robustez

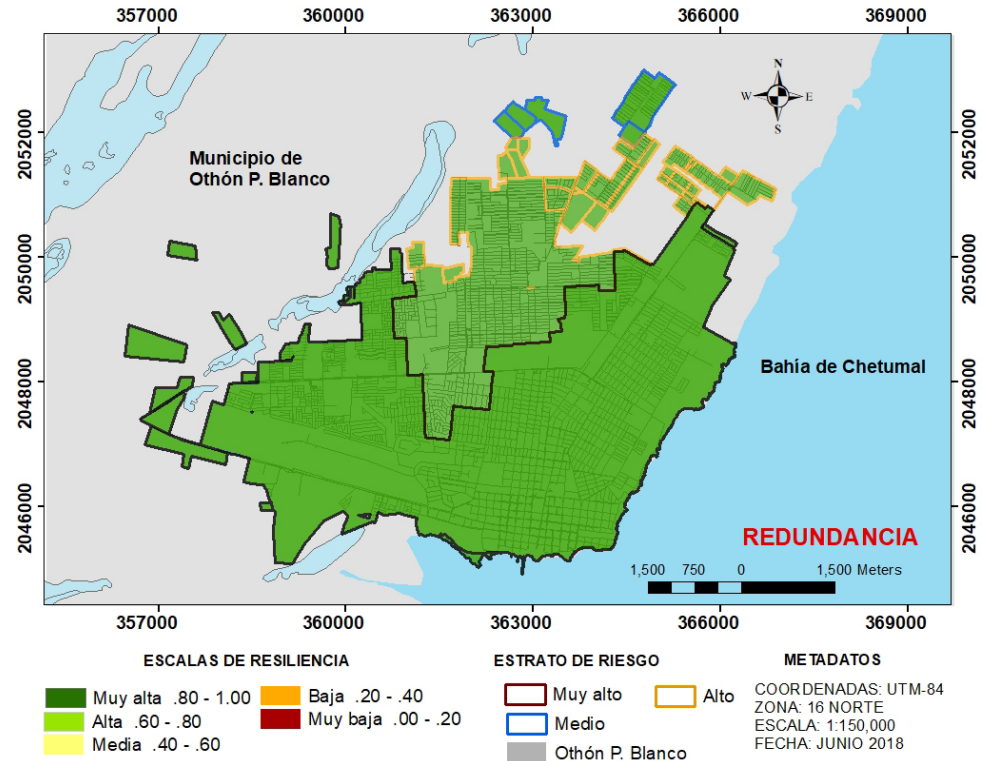

Fuente: elaboración propia

354 Revista Geográfica de América Central No 61E (3) Especial CLAG Este artículo está bajo una licencia e-ISSN 2215-2563 • Julio-diciembre 2018 • pp. 339-365 Atribución/Reconocimiento-NoCo Doi: http://dx.doi.org/10.15359/rgac.61-3.17 
Anita Martínez Méndez, Oscar Frausto Martínez, Lourdes Castillo Villanueva, José Manuel Camacho Sanabria. Índice de resiliencia de infraestructura de agua potable ante huracanes en ciudades costeras

Figura 7. Indicador recursos

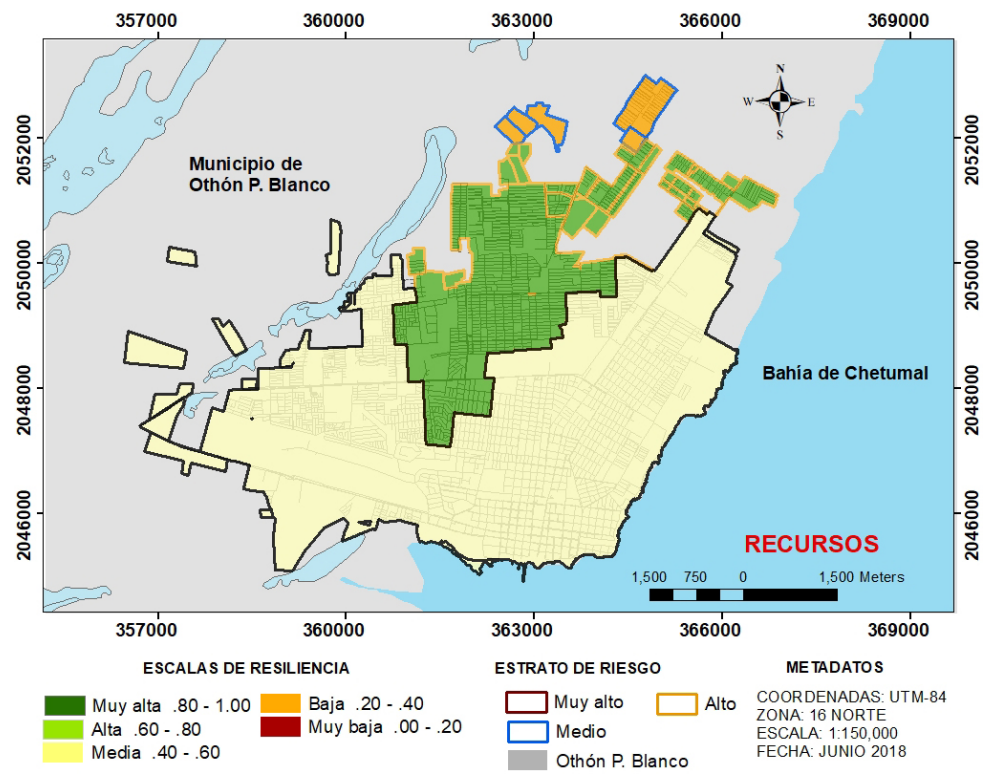

Fuente: elaboración propia

Por otra parte, el componente correspondiente a rapidez muestra una resiliencia muy alta en las tres zonas (figura 8). Eso indica que el sistema de agua potable, a pesar de sus vulnerabilidades en otros componentes, la reactivación en caso de un evento perturbador es rápida. El máximo de días mencionado por la población en reactivar el servicio cuando ha ocurrido un evento, fue de tres días, cabe señalar que solo el $15 \%$ de los entrevistados que proporcionaron esa respuesta.

En lo que respecta a la capacidad adaptativa del sistema sus valores oscilaron entre 0.50 y 0.72 (figura 9); correspondiendo el valor menos a la zona de peligro medio, esto indica que es el área con menor capacidad de adaptarse ante los efectos de un fenómeno perturbador. 
Anita Martínez Méndez, Oscar Frausto Martínez, Lourdes Castillo Villanueva, José Manuel Camacho Sanabria. Index of potable water infrastructure resilence facing hurricanes in coastal cities

Figura 8. Indicador rapidez

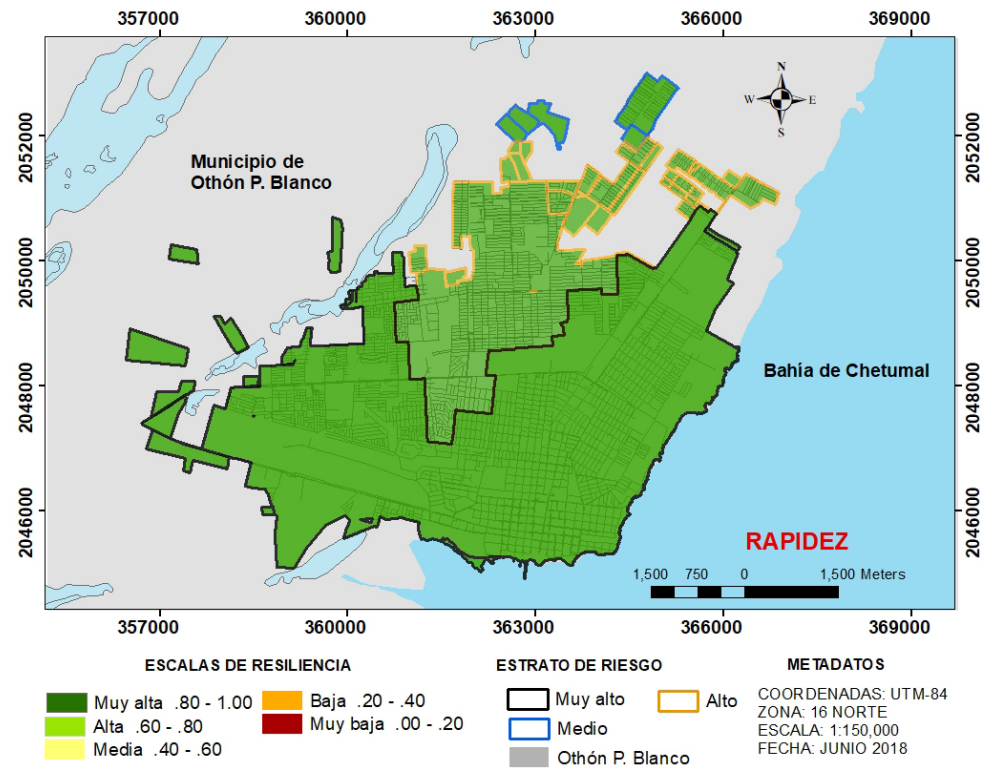

Fuente: elaboración propia

Figura 9. Indicador capacidad adaptativa

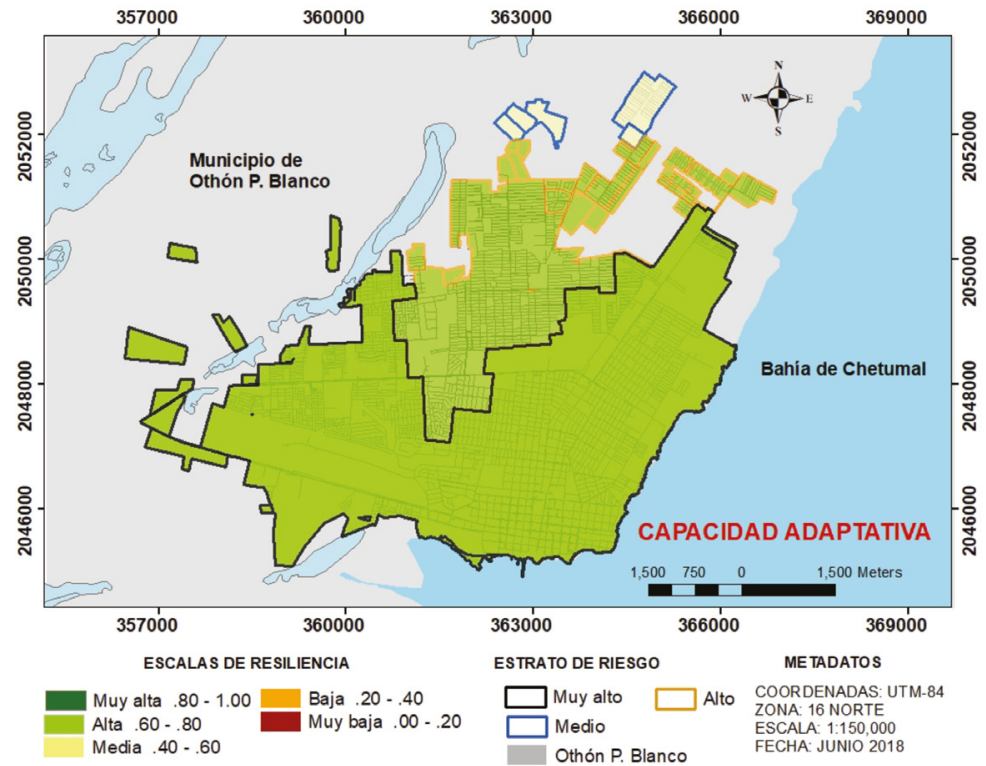

Fuente: elaboración propia

356 Revista Geográfica de América Central No 61E (3) Especial CLAG Este artículo está bajo una licencia e-ISSN 2215-2563 • Julio-diciembre 2018 • pp. 339-365 Atribución/Reconocimiento-NoComercialDoi: http://dx.doi.org/10.15359/rgac.61-3.17 
Anita Martínez Méndez, Oscar Frausto Martínez, Lourdes Castillo Villanueva, José Manuel Camacho Sanabria. Índice de resiliencia de infraestructura de agua potable ante huracanes en ciudades costeras

Comparando las tres zonas el componente con el indicador más bajo es la robustez con un promedio de 0.55 (resiliencia media), seguidamente el de recursos con un 0.59 (resiliencia medía), los restantes se encuentran entre 0.60 y 0.9 .4 presentando una resiliencia alta y muy alta.

Tabla 5. Resultados de índice de resiliencia del sistema de infraestructura de agua potable de Chetumal, México.

\begin{tabular}{|l|c|c|c|c|}
\hline \multirow{2}{*}{ COMPONENTE } & \multicolumn{3}{|c|}{ ESTRATOS (ZONAS) } & \multirow{2}{*}{$\begin{array}{c}\text { INDICADOR/ } \\
\text { ÍNDICE }\end{array}$} \\
\cline { 2 - 5 } & $\begin{array}{c}\text { PELIGRO MUY } \\
\text { ALTO }\end{array}$ & $\begin{array}{c}\text { PELIGRO } \\
\text { ALTO }\end{array}$ & $\begin{array}{c}\text { PELIGRO } \\
\text { MEDIO }\end{array}$ & \begin{tabular}{c} 
INO \\
\hline Robustez
\end{tabular} \\
\hline Redundancia & 0.17 & 0.16 & 0.17 & 0.17 \\
\hline Recursos & 0.12 & 0.26 & 0.24 & 0.25 \\
\hline Rapidez & 0.10 & 0.16 & 0.07 & 0.12 \\
\hline Capacidad adaptativa & 0.07 & 0.06 & 0.05 & 0.09 \\
\hline $\begin{array}{l}\text { Índice de resiliencia } \\
\text { del sistema } \\
\text { urbano costero de } \\
\text { infraestructura de } \\
\text { agua potable }\end{array}$ & $\mathbf{0 . 7 1}$ & $\mathbf{0 . 7 4}$ & $\mathbf{0 . 6 2}$ & $\mathbf{0 . 6 9}$ \\
\hline
\end{tabular}

Fuente: elaboración propia

El índice de resiliencia correspondiente a la zona con peligro muy alto se encuentra ubicada en el área costera (figura 7), presenta una resiliencia alta con una valor de 0.71 . El área con peligro alto presentó un índice de resiliencia del sistema de 0.74 , que indica la posibilidad de resiliencia alta. La zona correspondiente a peligro medio, ubicada al norte de la ciudad formada por los nuevos fraccionamientos (menor de 10 años), presenta un índice con valor de 0.62 que corresponde a la categoría de resiliencia alta. 
Figura 10. Índice de resiliencia del sistema de infraestructura de agua potable

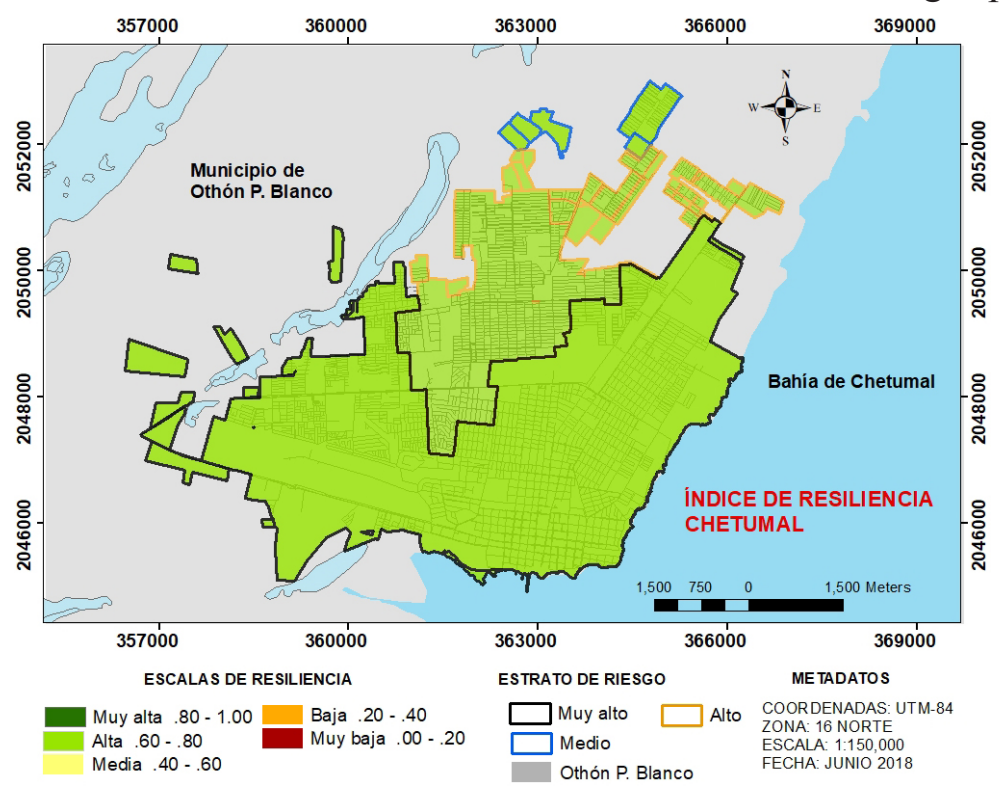

Fuente: elaboración propia

Las tres zonas de acuerdo a sus índices se ubicaron en la categoría de resiliencia alta en el sistema. Sin embargo, haciendo una comparación, se observa que el área correspondiente a peligro medio es la que registra el índice más bajo de las tres (0.062). Se podría inferir que lo anterior se debe a que son colonias nuevas y que algunos hogares no cuentan con el servicio de agua potable; además de que es población que no tiene la experiencia de haber experimentado un huracán en su vivienda actual, por lo tanto no tiene conocimiento sobre que afectaciones puede tener la infraestructura, siendo esto una variable considerada como positiva hacia la resiliencia. Asimismo, que un $38 \%$ de las viviendas no cuenta con algún método para mitigar la falta del servicio de agua potable, como tampoco cuentan con la alternativa de abastecerse de otra zona, lo que influye directamente en el componente de redundancia del sistema.

Las zonas correspondientes a peligro muy alto y alto el valor del índice es similar, estas dos zonas corresponden a los primeros dos cuadros de la ciudad, en los cuales se ha tenido avance en la sustitución de tuberías que tenían más de 30 años en funcionamiento, las cuales provocaban 
Anita Martínez Méndez, Oscar Frausto Martínez, Lourdes Castillo Villanueva, José Manuel Camacho Sanabria. Índice de resiliencia de infraestructura de agua potable ante huracanes en ciudades costeras

pérdidas considerables del vital líquido. Cabe señalar que en esa área a pesar de que la percepción de la población es de una afectación alta en el sistema, un $66 \%$ de ella cuentan conocimiento de lo que se debe hacer en caso de un huracán. A ello, se añade que en ellas se ubica la zona centro de la ciudad y la zona costera, siendo estos motivos de mayor atención por parte de las autoridades.

\section{Conclusiones}

Con base a la presente investigación se concluye que el enfoque de resiliencia en infraestructura puede considerarse como una alternativa para la construcción de sistemas los cuales tengan la capacidad hacer frente a las posibles perturbaciones de eventos (naturales y antropogénicos), o en su caso adaptarse. Una infraestructura resiliente puede considerarse un método preventivo ante desastres, toda vez que se centra en la capacidad de hacer frente y adaptarse ante eventos perturbadores.

El índice de resiliencia permitió la identificación de las áreas que presentan mayor vulnerabilidad en el sistema de infraestructura de agua potable. Por su parte, la zona correspondiente a peligro medio presenta la resiliencia más baja (0.62), lo que indica que es una zona que requiere mayor atención, con la finalidad de mejorar su estado y con ello su resiliencia. Asimismo, esto permitió identificar cuál de los cinco componentes presenta mayor vulnerabilidad, que en este caso, fue el de capacidad adaptativa con un valor de 0.62 encontrándose a 0.02 de presentar una resiliencia baja.

Dentro de las variables con influencia negativa se tuvieron siete, las cuales presentaron porcentajes considerables; por ejemplo las zonas con infraestructura mayor a 30 años son los dos primeros estratos de la ciudad que representa aproximadamente un $65 \%$ del total de la red. Por otra parte como bien se mencionó en la distribución de estratos, de acuerdo a la variable peligro ante huracanes, la ciudad se encuentra entre peligro alto y medio, y por ende, toda su infraestructura.

Por otra parte, una variable con influencia positiva, pero que tuvo incidencia negativa en la resiliencia; es que no existen fondos preventivos de atención a los sistemas. Únicamente a nivel federal se cuenta con el fondo de atención a emergencias, el cual es específicamente destinado para atender los impactos de fenómenos hidrometeorológicos, dentro de los que se encuentran los huracanes. A nivel estatal, existe un fondo destinado al 
mantenimiento de equipos y de las zonas de capitación, las cuales son atendidas permanentemente. Sin embargo para mantenimiento de la red no existe. Las fallas y vulnerabilidades del sistema, son atendidas hasta que se reportan por los usuarios (por ejemplo fugas), o en su caso que se haga evidente la rotura de una tubería o impacte un evento.

Los resultados muestran la importancia de planificar el sistema de infraestructura de agua potable tanto en función de las dinámicas de crecimiento poblacional y urbanización. Los índices realizados, hacen evidente que el sistema presenta vulnerabilidades en sus componentes dentro de las que se pueden mencionar: las modificaciones en la red principal, bajas presiones en las zonas que se encuentran al norte y oeste de la ciudad que tienen menor de 15 años de haberse creado.

Finalmente, la metodología utilizada en este trabajo es un aporte para la construcción de índices de infraestructura de sistemas de agua potable en ciudades costeras, el cual permite identificar las zonas y componentes vulnerables. Por lo tanto, puede aplicarse a estudios de caso con enfoques similares, sin dejar de considerar las características particulares de cada espacio.

\section{Referencias}

Ahern, N. R., Kiehl, E. M., Sole, M. L., \& Byers, J. (2006). A Review of Instruments Measuring Resilience. Issues In Comprehensive Pediatric Nursing, 29(2), 103-125. doi:10.1080/01460860600677643

Azuz, I., Arredondo, M., Espejel, I., Rivera, E., Seingier, G. \& Ferman, J. (2010) Propuesta de indicadores de la Red Mexicana de Manejo Integrado Costero-Marino. En E. Rivera, I. Azuz, L. Alpuche \& G. Villalobos. (Eds). Cambio climático en México un enfoque costero y marino (pp. 901-939). Campeche: Universidad Autónoma de

Campeche, CETYS-Universidad y Gobierno del Estado de Campeche.

Bruneau, M., Chang, S. E., Eguchi, R. T., Lee, G. C., O'Rourke, T. D., Reinhorn, A. M. \& Von Winterfeldt, D. (2003). A framework to quantitatively assess and enhance the seismic resilience of communities.

Earthquake spectra, 19(4), 733-752.

Castillo-Villanueva, L., \& Velázquez-Torres, D. (2015). Sistemas complejos adaptativos, sistemas socio-ecológicos y resiliencia. Quivera, 17(2), 11-32. 
Anita Martínez Méndez, Oscar Frausto Martínez, Lourdes Castillo Villanueva, José Manuel Camacho Sanabria. Índice de resiliencia de infraestructura de agua potable ante huracanes en ciudades costeras

Comisión de Agua Potable y Alcantarillado (CAPA). (2015). Informe anual de actividades. Gobierno del estado de Quintana Roo.

Domínguez Serrano, Judith. (2010). El acceso al agua y saneamiento: Un problema de capacidad institucional local. Análisis en el estado de Veracruz. Gestión y política pública, 19(2), 311-350. Recuperado en 19 de junio de 2018, de http:/www.scielo.org.mx/scielo. php?script=sci_arttext\&pid=S1405-10792010000200004\&lng=es \&tlng=es.

Folke, C., S. R. Carpenter, B. Walker, M. Scheffer, T. Chapin, and J. Rockström. 2010. Resilience thinking: integrating resilience, adaptability and transformability. Ecology and Society 15(4): 20.

Frankenberg, E., Sikoki, B., Sumantri, C., Suriastini, W. \& Thomas, D. (2013). Education, vulnerability, and resilience after a natural disaster. Ecology and society: a journal of integrative science for resilience and sustainability, 18(2), 16.

Gay, A.L. (2016). Infraestructura resiliente: desempeño sostenido en un mundo siempre cambiante. Entretextos, $8 / 24$

Holling, C. S. (1973). Resilience and stability of ecological systems. Annual review of ecology and systematics, 4(1), 1-23.

Holling, C. S. (1996). Engineering resilience versus ecological resilience. Engineering within ecological constraints, 31(1996), 32.

Ilaya, A. (2015). Análisis de la reducción de la capacidad de la red y su relación con el suministro intermitente de agua. Los servicios de agua y drenaje con una visión integral. Guanajuato, Gto. México. Universidad de Guanajuato. (pp. 348-361)

INEGI. (2010). Porcentaje de viviendas particulares habitadas con disponibilidad de agua por entidad federativa, 1990 a 2010. Instituto Nacional de Estadística Geografía e Informática. México.

Leichenko, R. (2011). Climate change and urban resilience. Current opinion in environmental sustainability, 3(3), 164-168.

Labaka, L., Hernantes, J. \& Sarriegi J, (2015) "A framework to improve the resilience of critical infrastructures", International Journal of Disaster Resilience in the Built Environment, Vol. 6 Issue: 4, pp.409423, https://doi.org/10.1108/IJDRBE-07-2014-0048 
O’Rourke, T. D., Jeon, S. S., Eguchi, R. T., \& Huyck, C. K. (2001). Advanced GIS for loss estimation and rapid post-earthquake assessment of building damage. Research Progress and Accomplishment, 157-164. O'Rourke, T. D. (2007). Critical infrastructure, interdependencies, and resilience. BRIDGE-Washington-National Academy of Engineering-, $37(1), 22$.

Sánchez, et al. (2015). Caracterización hidrogeoquímica de las aguas subterráneas del

Schultz, M. T., \& Smith, E. R. (2016). Assessing the Resilience of Coastal Systems: A Probabilistic Approach. Journal Of Coastal Research, 32(5), 1032-1050. doi:10.2112/JCOASTRES-D-15-00170.1

Seingier, G., Espejel, I., Fermán, J. \& Delgado, O. (2010). Vulnerabilidad de las poblaciones costeras ante la peligrosidad natural, enfoque estatal y municipal. En E. Rivera, I. Azuz, L. Alpuche \& G. Villalobos. (Eds). Cambio climático en México un enfoque costero y marino (pp. 669-688). Campeche: Universidad Autónoma de Campeche, CETYSUniversidad y Gobierno del Estado de Campeche.

Shinozuka, M., Chang, S. E., Cheng, T. C., Feng, M., O'Rourke, T. D., Saadeghvaziri, M. A. \& Shi, P. (2004). Resilience of integrated power and water systems. Seismic Evaluation and Retrofit of Lifeline Systems, Articles from MCEER's Research Progress and Accomplishments Volumes, 65-86.

Tamvakis, P., \& Xenidis, Y. (2013). Comparative evaluation of resilience quantification methods for infrastructure systems. Procedia-Social and Behavioral Sciences, 74, 339-348. Recuperado de: https://doi. org/10.1016/j.sbspro.2013.03.030

Vázquez Sánchez, Martín; Méndez Ramírez, José Juan y Mastachi Loza, Carlos Alberto (2016): índice de resiliencia urbana en el abastecimiento del agua potable en Lerma y San Mateo Atenco, estado de México. In: El desarrollo regional frente al cambio ambiental global y la transición hacia la sustentabilidad. Asociación Mexicana de Ciencias para el Desarrollo Regional A.C., México. ISBN AMECIDER: 978-607-96649-2-3 UNAM: 978-607-02-8564-

Walker, B., Holling, C. S., Carpenter, S., \& Kinzig, A. (2004). Resilience, adaptability and transformability in social-ecological systems. Ecology and society, 9(2). 
Anita Martínez Méndez, Oscar Frausto Martínez, Lourdes Castillo Villanueva, José Manuel Camacho Sanabria. Índice de resiliencia de infraestructura de agua potable ante huracanes en ciudades costeras

Windle, G., Bennett, K. M., \& Noyes, J. (2011). A methodological review of resilience measurement scales. Health \& Quality Of Life Outcomes, 9(1), 8-25. doi:10.1186/1477-7525-9-8

Yáñez, A., Way, J., Jacob, J., Ibáñez, C., Martínez, A., Miranda, A., Tejeda,A., Welsh, C. \& Carranza, A. (2010). Panel INECOL 2008 -Conclusiones- La zona costera y sus impactos ecológicos, económicos y sociales. En A. Yáñez (Ed). Impactos del cambio climático sobre la zona costera (pp. 173-179). México: Instituto Nacional de Ecología INE-SEMARNAT e Instituto de Ecología A. C. INECOL

Zhou, H., Wan, J., \& Jia, H. (2010). Resilience to natural hazards: a geographic perspective. Natural Hazards, 53(1), 21-41 
Anita Martínez Méndez, Oscar Frausto Martínez, Lourdes Castillo Villanueva, José Manuel Camacho Sanabria. Index of potable water infrastructure resilence facing hurricanes in coastal cities

Anexo 1. Ponderación indicadores

\begin{tabular}{|c|c|c|c|}
\hline COMPONENTE & INDICADOR & CLASIFICACIÓN & $\begin{array}{l}\text { PONDERACIÓN } \\
\text { POR EL EXPERTO }\end{array}$ \\
\hline \multirow[t]{7}{*}{ Resistencia } & $\begin{array}{l}\text { Zonas con afectaciones en la } \\
\text { infraestructura de agua potable }\end{array}$ & Negativo & 20 \\
\hline & $\begin{array}{l}\text { Zonas con mas de } 30 \text { con la } \\
\text { infraestructura }\end{array}$ & Negativo & 30 \\
\hline & $\begin{array}{l}\text { Careamos ubicados en zonas de } \\
\text { riesgo alto }\end{array}$ & Negativo & 20 \\
\hline & $\begin{array}{l}\text { Zonas con tubería de polietileno de } \\
\text { alta densidad (PAD) }\end{array}$ & Positivo & 10 \\
\hline & Ley de obra pública & Positivo & 5 \\
\hline & Reglamentos de construcción & Positivo & 5 \\
\hline & $\begin{array}{l}\text { Manuales de construcción para } \\
\text { infraestructura de zonas costeras }\end{array}$ & Positivo & 10 \\
\hline \multirow[t]{10}{*}{ Redundancia } & $\begin{array}{l}\text { Número de obras de infraestructura } \\
\text { de nueva creación }\end{array}$ & Positivo & 5 \\
\hline & $\begin{array}{l}\text { Población que desconoce que } \\
\text { hacer en caso de huracán (para su } \\
\text { infraestructura) }\end{array}$ & Negativo & 5 \\
\hline & $\begin{array}{l}\text { Población que puede abastecerse de } \\
\text { otro sector de la ciudad }\end{array}$ & Positivo & 5 \\
\hline & $\begin{array}{l}\text { Planes de mantenimiento de } \\
\text { infraestructura }\end{array}$ & Positivo & 20 \\
\hline & Zonas con presión bala & Negativo & 10 \\
\hline & Número de usuarios & Negativo & 5 \\
\hline & Número de energías alternas & Negativo & 20 \\
\hline & $\begin{array}{l}\text { Plan de emergencias en caso de } \\
\text { huracán a nivel federal }\end{array}$ & Positivo & 10 \\
\hline & $\begin{array}{l}\text { Plan de emergencias en caso de } \\
\text { huracán a nivel estatal }\end{array}$ & Positivo & 10 \\
\hline & $\begin{array}{l}\text { Plan de emergencias en caso de } \\
\text { huracán a nivel municipal }\end{array}$ & Positivo & 10 \\
\hline \multirow[t]{4}{*}{ Recursos } & $\begin{array}{l}\text { Población que invierte en la } \\
\text { recuperación de la infraestructura } \\
\text { después de un evento }\end{array}$ & Positivo & 10 \\
\hline & $\begin{array}{l}\text { Fondos considerados previo al } \\
\text { impacto de un huracán (federal) }\end{array}$ & Positivo & 40 \\
\hline & $\begin{array}{l}\text { Fondos considerados previo al } \\
\text { impacto de un huracán (estatal) }\end{array}$ & Positivo & 40 \\
\hline & $\begin{array}{l}\text { Fondos considerados previo al } \\
\text { impacto de un huracán (municipal) }\end{array}$ & Positivo & 10 \\
\hline
\end{tabular}


Anita Martínez Méndez, Oscar Frausto Martínez, Lourdes Castillo Villanueva, José Manuel Camacho Sanabria. Índice de resiliencia de infraestructura de agua potable ante huracanes en ciudades costeras

\begin{tabular}{|c|c|c|c|}
\hline COMPONENTE & INDICADOR & CLASIFICACIÓN & $\begin{array}{l}\text { PONDERACIÓN } \\
\text { POR EL EXPERTO }\end{array}$ \\
\hline \multirow[t]{3}{*}{$\begin{array}{l}\text { Rapidez (tiempo } \\
\text { de recuperación) }\end{array}$} & $\begin{array}{l}\text { Tiempo promedio en días de } \\
\text { reactivación del servicio }\end{array}$ & Positivo & 20 \\
\hline & $\begin{array}{l}\text { Capacitación al personal de la } \\
\text { dependencia normativa }\end{array}$ & Positivo & 40 \\
\hline & $\begin{array}{l}\text { Capacitación al personal del } \\
\text { organismo operador }\end{array}$ & Positivo & 40 \\
\hline \multirow[t]{8}{*}{$\begin{array}{l}\text { Capacidad } \\
\text { adaptativa }\end{array}$} & $\begin{array}{l}\text { Población con conocimiento sobre } \\
\text { que se debe hacer en caso de huracán }\end{array}$ & Positivo & 10 \\
\hline & $\begin{array}{l}\text { Población que participaría en caso de } \\
\text { afectación de huracán }\end{array}$ & Positivo & 10 \\
\hline & $\begin{array}{l}\text { Población con conocimiento sobre } \\
\text { los programas implementados por el } \\
\text { gobierno }\end{array}$ & Positivo & 15 \\
\hline & $\begin{array}{l}\text { Población que ha recibido } \\
\text { capacitación }\end{array}$ & Positivo & 10 \\
\hline & $\begin{array}{l}\text { Población que implementa métodos } \\
\text { para disminuir el impacto en la } \\
\text { infraestructura }\end{array}$ & Positivo & 10 \\
\hline & $\begin{array}{l}\text { Número de obras que se han } \\
\text { construido para disminuir el riesgo } \\
\text { ante huracanes }\end{array}$ & Positivo & 20 \\
\hline & $\begin{array}{l}\text { Población que ha vivido la } \\
\text { experiencia de un huracán }\end{array}$ & Positivo & 15 \\
\hline & $\begin{array}{l}\text { Población que conoce que es } \\
\text { resiliencia }\end{array}$ & Positivo & 10 \\
\hline
\end{tabular}


